This postprint is published in:

Colloquium mathematicum, Volume 149, Number 2, 2017, 211-224

DOI: $10.4064 / \mathrm{cm} 6785-4-2017$

\title{
CLASSIFYING HOMOGENEOUS CELLULAR ORDINAL BALLEANS UP TO COARSE EQUIVALENCE
}

\author{
T. BANAKH, I. PROTASOV, D. REPOVŠ, AND S. SLOBODIANIUK
}

\begin{abstract}
For every ballean $X$ we introduce two cardinal characteristics $\operatorname{cov}^{b}(X)$ and $\operatorname{cov}^{\sharp}(X)$ describing the capacity of balls in $X$. We observe that these cardinal characteristics are invariant under coarse equivalence and prove that two cellular ordinal balleans $X, Y$ are coarsely equivalent if $\operatorname{cof}(X)=\operatorname{cof}(Y)$ and $\operatorname{cov}^{b}(X)=\operatorname{cov}^{\sharp}(X)=\operatorname{cov}^{b}(Y)=$ $\operatorname{cov}^{\sharp}(Y)$. This result implies that a cellular ordinal ballean $X$ is homogeneous if and only if $\operatorname{cov}^{b}(X)=\operatorname{cov}^{\sharp}(X)$. Moreover, two homogeneous cellular ordinal balleans $X, Y$ are coarsely equivalent if and only if $\operatorname{cof}(X)=\operatorname{cof}(Y)$ and $\operatorname{cov}^{\sharp}(X)=\operatorname{cov}^{\sharp}(Y)$ if and only if each of these balleans coarsely embeds into the other ballean. This means that the coarse structure of a homogeneous cellular ordinal ballean $X$ is fully determined by the values of the cardinals $\operatorname{cof}(X)$ and $\operatorname{cov}^{\sharp}(X)$. For every limit ordinal $\gamma$ we shall define a ballean $2^{<\gamma}$ (called the Cantor macro-cube), which in the class of cellular ordinal balleans of cofinality $\operatorname{cf}(\gamma)$ plays a role analogous to the role of the Cantor cube $2^{\kappa}$ in the class of zero-dimensional compact Hausdorff spaces. We shall also present a characterization of balleans which are coarsely equivalent to $2^{<\gamma}$. This characterization can be considered as an asymptotic analogue of Brouwer's characterization of the Cantor cube $2^{\omega}$.
\end{abstract}

\section{INTRODUCTION}

In this paper we study the structure of ordinal balleans, i.e., balleans that have well-ordered base of their coarse structure. Such balleans were introduced by Protasov in [10]. Some basic facts about ordinal balleans are discussed in Section 1. The main result of the paper is presented in Section 2 containing a criterion for recognizing coarsely equivalent cellular ordinal balleans. In Section 3 we shall use this criterion to classify homogeneous cellular ordinal balleans up to coarse equivalence. In Section 4 we apply this criterion to characterize balleans $2^{<\gamma}$ (called Cantor macro-cubes), which are universal objects in the class of cellular ordinal balleans. In Section 4 also we identify the natural coarse structure on additively indecomposable ordinals.

2010 Mathematics Subject Classification. 54E35; 51F99.

Key words and phrases. Coarse space, ballean, cellular ballean, ordinal ballean, homogeneous ballean, coarse equivalence, cellular entourage, asymptotic dimension, Cantor macro-cube. 


\section{Ordinal Balleans}

The notion of a ballean was introduced by Protasov [11] as a large scale counterpart of a uniform space and is a modification of the notion of a coarse space introduced by Roe [14]. Both notions are defined as sets endowed with certain families of entourages.

By an entourage on a set $X$ we understand any reflexive symmetric relation $\varepsilon \subset X \times X$. This means that $\varepsilon$ contains the diagonal $\Delta_{X}=\{(x, y) \in$ $X \times X: x=y\}$ of $X \times X$ and is symmetric in the sense that $\varepsilon=\varepsilon^{-1}$ where $\varepsilon^{-1}=\{(y, x) \in X \times X:(x, y) \in \varepsilon\}$. An entourage $\varepsilon \subset X \times X$ will be called cellular if it is transitive, i.e., it is an equivalence relation on $X$.

Each entourage $\varepsilon \subset X \times X$ determines a cover $\{B(x, \varepsilon): x \in X\}$ of $X$ by $\varepsilon$ balls $B(x, \varepsilon)=\{y \in X:(x, y) \in \varepsilon\}$. It follows that $\varepsilon=\bigcup_{x \in X}\{x\} \times B(x, \varepsilon)=$ $\bigcup_{x \in X} B(x, \varepsilon) \times\{x\}$, so the entourage $\varepsilon$ can be fully recovered from the system of balls $\{B(x, \varepsilon): x \in \varepsilon\}$. For a subset $A \subset X$ we let $B(A, \varepsilon)=\bigcup_{a \in A} B(a, \varepsilon)$ denote the $\varepsilon$-neighborhood of $A$.

A ballean is a pair $\left(X, \mathcal{E}_{X}\right)$ consisting of a set $X$ and a family $\mathcal{E}_{X}$ of entourages on $X$ (called the set of radii) such that $\bigcup \mathcal{E}_{X}=X \times X$ and for any entourages $\varepsilon, \delta \in \mathcal{E}_{X}$ their composition

$$
\varepsilon \circ \delta=\{(x, z) \in X \times X: \exists y \in X(x, y) \in \varepsilon,(y, z) \in \delta\}
$$

is contained in some entourage $\eta \in \mathcal{E}_{X}$. A ballean $\left(X, \mathcal{E}_{X}\right)$ is called a coarse space if the family $\mathcal{E}_{X}$ is closed under taking subentourages, i.e., for any $\varepsilon \in \mathcal{E}_{X}$ any entourage $\delta \subset \varepsilon$ belongs to $\mathcal{E}_{X}$. In this case the set of radii $\mathcal{E}_{X}$ is called a coarse structure on $X$. Each set of radii $\mathcal{E}_{X}$ can be completes to the coarse structure $\downarrow \mathcal{E}_{X}$ consisting of all possible subentourages $\delta \subset \varepsilon \in \mathcal{E}_{X}$. In this case $\mathcal{E}_{X}$ is called a base of the coarse structure $\downarrow \mathcal{E}_{X}$. So, balleans can be considered as coarse spaces with a fixed base of their coarse structure. Coarse spaces and coarse structures were introduced by Roe [14].

Each subset $A \subset X$ of a ballean $\left(X, \mathcal{E}_{X}\right)$ carries the induced ballean structure $\mathcal{E}_{A}=\left\{\varepsilon \cap A^{2}: \varepsilon \in \mathcal{E}_{X}\right\}$. The ballean $\left(A, \mathcal{E}_{A}\right)$ will be called a subballean of $\left(X, \mathcal{E}_{X}\right)$.

Now we consider some examples of balleans.

Example 1.1. Each metric space $(X, d)$ carries a canonical ballean structure $\mathcal{E}_{X}=\left\{\Delta_{\varepsilon}\right\}_{\varepsilon \in \mathbb{R}_{+}}$consisting of the entourages

$$
\Delta_{\varepsilon}=\{(x, y) \in X \times X: d(x, y) \leq \varepsilon\}
$$

parametrized by the set $\mathbb{R}_{+}=[0, \infty)$ of non-negative real numbers. The ballean structure $\mathcal{E}_{X}=\left\{\Delta_{\varepsilon}\right\}_{\varepsilon \in \mathbb{R}_{+}}$generates the coarse structure $\downarrow \mathcal{E}_{X}$ consisting of all subentourages of the entourages $\Delta_{\varepsilon}, \varepsilon \in \mathbb{R}_{+}$.

A ballean $X$ is called metrizable if its coarse structure is generated by a suitable metric. Metrizable balleans belong to the class of ordinal balleans. A ballean $\mathbf{X}=\left(X, \mathcal{E}_{X}\right)$ is defined to be ordinal if its coarse structure $\downarrow \mathcal{E}_{X}$ has a well-ordered base $\mathcal{B} \subset \mathcal{E}_{X}$. The latter means that $\mathcal{B}$ can be enumerated as $\left\{B_{\alpha}\right\}_{\alpha<\kappa}$ for some ordinal $\kappa$ such that $B_{\alpha} \subset B_{\beta}$ for all ordinals $\alpha<\beta<\kappa$. 
Passing to a cofinal subset of $\kappa$, we can always assume that $\kappa$ is a regular cardinal, equal of the cofinality $\operatorname{cof}(\mathbf{X})$. By definition, the cofinality $\operatorname{cof}(\mathbf{X})$ of a ballean $\mathbf{X}=\left(X, \mathcal{E}_{X}\right)$ is equal to the smallest cardinality of a base of the coarse structure $\downarrow \mathcal{E}_{X}$.

Ordinal balleans can be characterized as balleans $\mathbf{X}=\left(X, \mathcal{E}_{X}\right)$ whose cofinality equals the additivity number

$$
\operatorname{add}(\mathbf{X})=\min \left\{|\mathcal{A}|: \mathcal{A} \subset \downarrow \mathcal{E}_{X}, \bigcup \mathcal{A} \notin \downarrow \mathcal{E}_{X} \backslash\{X \times X\}\right\} .
$$

Proposition 1.2. A ballean $X$ is ordinal if and only if $\operatorname{cof}(X)=\operatorname{add}(X)$.

Proof. Assuming that a ballean $\left(X, \mathcal{E}_{X}\right)$ is ordinal, fix a well-ordered base $\left\{\varepsilon_{\alpha}\right\}_{\alpha<\kappa}$ of the coarse structure $\downarrow \mathcal{E}_{X}$ of $\mathcal{E}_{X}$. Passing to a cofinal subsequence, we can assume that $\kappa=\operatorname{cf}(\kappa)$ is a regular cardinal. If $\kappa=1$, then the ballean $\left(X, \mathcal{E}_{X}\right)$ is bounded and hence for the entourage $X \times X \in \downarrow \mathcal{E}_{X}$ the family $\mathcal{A}=\{X \times X\}$ has cardinality $|\mathcal{A}|=1$ and $\bigcup \mathcal{A}=X \times X \notin \mathcal{E}_{X} \backslash\{X \times X\}$. Therefore, $\operatorname{add}(X)=1=\operatorname{cof}(X)$. So, we assume that the regular cardinal $\kappa$ is infinite and hence $\varepsilon_{\alpha} \neq X \times X$ for all $\alpha<\kappa$. Since $\operatorname{add}(X) \leq \operatorname{cof}(X)$, it suffices to check that $\operatorname{cof}(X) \leq \operatorname{add}(X)$. The definition of the cardinal $\operatorname{cof}(X)$ implies that $\operatorname{cof}(X) \leq \kappa$. The inequality $\operatorname{add}(X) \geq \kappa \geq \operatorname{cof}(X)$ will follow as soon as we check that for any family $\mathcal{A} \subset \mathcal{E}_{X}$ of cardinality $|\mathcal{A}|<\kappa$ we get $\bigcup \mathcal{A} \in \downarrow \mathcal{E}_{X} \backslash\{X \times X\}$. For every set $A \in \mathcal{A}$ find an ordinal $\alpha_{A}<\kappa$ such that $A \subset \varepsilon_{\alpha_{A}}$. By the regularity of the cardinal $\kappa$, the cardinal $\beta=\sup \left\{\alpha_{A}: A \in \mathcal{A}\right\}$ is strictly smaller than $\kappa$. Consequently, $A \subset \varepsilon_{\alpha_{A}} \subset \varepsilon_{\beta}$ for every $A \in \mathcal{A}$ and hence $\bigcup \mathcal{A} \subset \varepsilon_{\beta}$ and $\bigcup \mathcal{A} \in \downarrow \mathcal{E}_{X} \backslash\{X \times X\}$. This completes the proof of the equality $\operatorname{add}(X)=\operatorname{cof}(X)$ for ordinal balleans.

Now we shall prove that a ballean $\left(X, \mathcal{E}_{X}\right)$ is ordinal if $\operatorname{add}(X)=\operatorname{cof}(X)$. Fix any base $\left\{\varepsilon_{\alpha}\right\}_{\alpha<\operatorname{cof}(X)} \subset \downarrow \mathcal{E}_{X}$ of the coarse structure $\downarrow \mathcal{E}_{X}$ of $X$. By definition of the additivity number $\operatorname{add}(X)$, for every $\alpha<\operatorname{cof}(X)=\operatorname{add}(X)$, the union $\tilde{\varepsilon}_{\alpha}=\bigcup_{\beta \leq \alpha} \varepsilon_{\beta}$ belongs to the coarse structure $\downarrow \mathcal{E}_{X}$. Then $\left(\tilde{\varepsilon}_{\alpha}\right)_{\alpha<\operatorname{cof}(X)}$ is a well-ordered base of the coarse structure $\downarrow \mathcal{E}_{X}$, which means that the ballean $\left(X, \mathcal{E}_{X}\right)$ is ordinal.

An important property of ordinal balleans of uncountable cofinality is their cellularity. A ballean $\left(X, \mathcal{E}_{X}\right)$ is called cellular if its coarse structure $\downarrow \mathcal{E}_{X}$ has a base consisting of cellular entourages (i.e., equivalence relations). It can be shown that a ballean $\left(X, \mathcal{E}_{X}\right)$ is cellular if and only if for every $\varepsilon \in \mathcal{E}_{X}$ the cellular entourage $\varepsilon^{<\omega}=\bigcup_{n \in \omega} \varepsilon^{n}$ belongs to the coarse structure $\downarrow \mathcal{E}_{X}$. Here $\varepsilon^{0}=\Delta_{X}$ and $\varepsilon^{n+1}=\varepsilon^{n} \circ \varepsilon$ for all $n \in \omega$. This characterization implies the following simple fact.

Proposition 1.3. Each ordinal ballean $\mathbf{X}=\left(X, \mathcal{E}_{X}\right)$ with uncountable cofinality $\operatorname{cof}(\mathbf{X})$ is cellular.

Remark 1.4. By [13, Theorem 3.1.3], a ballean $X$ is cellular if and only if it has asymptotic dimension $\operatorname{asdim}(X)=0$. A metrizable ballean $X$ is cellular if and only if its coarse structure is generated by an ultrametric (i.e., a metric $d$ satisfying the strong triangle inequality $d(x, z) \leq \max \{d(x, y), d(y, z)\}$ for 
all point $x, y, z \in X)$. More information on cellular balleans can be found in [13, Chapter 3]. For information on space of asymptotic dimension zero, see $[4]$.

Example 1.5. Every infinite cardinal $\kappa$ carries a natural ballean structure $\mathcal{E}_{\kappa}=\left\{\varepsilon_{\alpha}\right\}_{\alpha<\kappa}$ consisting of the entourages

$$
\varepsilon_{\alpha}=\{(x, y) \in \kappa \times \kappa: x \leq y+\alpha, y \leq x+\alpha\}
$$

parametrized by ordinals $\alpha<\kappa$. The obtained ordinal ballean $\left(\kappa, \mathcal{E}_{\kappa}\right)$ will be denoted by $\overleftrightarrow{\kappa}$. Cardinal balleans $\overleftrightarrow{\kappa}$ were introduced in [8]. By Theorem 3 of [8], the ballean $\overleftrightarrow{\kappa}$ is cellular for any uncountable cardinal $\kappa$.

Example 1.6. Given an ordinal $\gamma$ and a transfinite sequence $\left(\kappa_{\alpha}\right)_{\alpha<\gamma}$ of non-zero cardinals, consider the ballean

$$
\coprod_{\alpha \in \gamma} \kappa_{\alpha}=\left\{\left(x_{\alpha}\right)_{\alpha \in \gamma} \in \prod_{\alpha \in \gamma} \kappa_{\alpha}:\left|\left\{\alpha \in \gamma: x_{\alpha} \neq 0\right\}\right|<\omega\right\}
$$

endowed with the ballean structure $\left\{\varepsilon_{\beta}\right\}_{\beta<\gamma}$ consisting of the entourages

$$
\varepsilon_{\beta}=\left\{\left(\left(x_{\alpha}\right)_{\alpha \in \gamma},\left(y_{\alpha}\right)_{\alpha \in \gamma}\right) \in\left(\coprod_{\alpha<\gamma} \kappa_{\alpha}\right)^{2}: \forall \alpha>\beta\left(x_{\alpha}=y_{\alpha}\right)\right\} \text { for } \beta<\gamma .
$$

The ballean $\coprod_{\alpha \in \gamma} \kappa_{\alpha}$ is called the asymptotic product of cardinals $\kappa_{\alpha}, \alpha \in \gamma$. It is a cellular ordinal ballean whose cofinality equals $\operatorname{cf}(\gamma)$, the cofinality of the ordinal $\gamma$.

If all cardinals $\kappa_{\alpha}, \alpha \in \gamma$, are equal to a fixed cardinal $\kappa$, then the asymptotic product $\coprod_{\alpha \in \gamma} \kappa_{\alpha}$ will be denoted by $\kappa^{<\gamma}$. For a limit ordinal $\gamma$ the ballean $2^{<\gamma}$ is called a Cantor macro-cube. The Cantor macro-cube $2^{<\omega}$ was characterized in [3]. This characterization will be extended to all Cantor macro-cubes in Theorem 4.3.

Balleans are objects of the (coarse) category whose morphisms are coarse maps. A map $f: X \rightarrow Y$ between two balleans $\left(X, \mathcal{E}_{X}\right)$ and $\left(Y, \mathcal{E}_{Y}\right)$ is called coarse if for each $\varepsilon \in \mathcal{E}_{X}$ there is $\delta \in \mathcal{E}_{Y}$ such that $\{(f(x), f(y)):(x, y) \in$ $\varepsilon\} \subset \delta$. A map $f: X \rightarrow Y$ is called a coarse isomorphism if $f$ is bijective and both maps $f$ and $f^{-1}$ are coarse. In this case the balleans $\left(X, \mathcal{E}_{X}\right)$ and $\left(Y, \mathcal{E}_{Y}\right)$ are called coarsely isomorphic. It follows that each ballean $\left(X, \mathcal{E}_{X}\right)$ is coarsely isomorphic to the coarse space $\left(X, \downarrow \mathcal{E}_{X}\right)$.

Coarse isomorphisms play the role of isomorphisms in the coarse category (whose objects are balleans and morphisms are coarse maps). Probably a more important notion is that of a coarse equivalence of balleans. Two balleans $\left(X, \mathcal{E}_{X}\right)$ and $\left(Y, \mathcal{E}_{Y}\right)$ are coarsely equivalent if they contain coarsely isomorphic large subspaces $L_{X} \subset X$ and $L_{Y} \subset Y$. A subset $L$ of a ballean $\left(X, \mathcal{E}_{X}\right)$ is called large if $X=B(L, \varepsilon)$ for some entourage $\varepsilon \in \mathcal{E}_{X}$.

Coarse equivalences can be alternatively defined using multi-valued maps. By a multi-valued map (briefly, a multi-map) $\Phi: X \multimap Y$ between two sets $X, Y$ we understand any subset $\Phi \subset X \times Y$. For a subset $A \subset X$ by 
$\Phi(A)=\{y \in Y: \exists a \in A$ with $(a, y) \in \Phi\}$ we denote the image of $A$ under the multi-map $\Phi$. Given a point $x \in X$ we write $\Phi(x)$ instead of $\Phi(\{x\})$.

The inverse $\Phi^{-1}: Y \multimap X$ of the multi-map $\Phi: X \multimap Y$ is the multi-map

$$
\Phi^{-1}=\{(y, x) \in Y \times X:(x, y) \in \Phi\} \subset Y \times X
$$

assigning to each point $y \in Y$ the set $\Phi^{-1}(y)=\{x \in X: y \in \Phi(x)\}$. For two multi-maps $\Phi: X \multimap Y$ and $\Psi: Y \multimap Z$ we define their composition $\Psi \circ \Phi: X \multimap Z$ as usual:

$\Psi \circ \Phi=\{(x, z) \in X \times Z: \exists y \in Y$ such that $(x, y) \in \Phi$ and $(y, z) \in \Psi\}$.

A multi-map $\Phi: X \multimap Y$ between two balleans $\left(X, \mathcal{E}_{X}\right)$ and $\left(Y, \mathcal{E}_{Y}\right)$ is called coarse if for every $\varepsilon \in \mathcal{E}_{X}$ there is an entourage $\delta \in \mathcal{E}_{Y}$ containing the set $\omega_{\Phi}(\varepsilon)=\bigcup_{(x, y) \in \varepsilon} \Phi(x) \times \Phi(y)$ called the $\varepsilon$-oscillation of $\Phi$. More precisely, for a function $\varphi: \mathcal{E}_{X} \rightarrow \mathcal{E}_{Y}$ a multi-map $\Phi: X \multimap Y$ is defined to be $\varphi$-coarse if $\omega_{\Phi}(\varepsilon) \subset \varphi(\varepsilon)$ for every $\varepsilon \in \mathcal{E}_{X}$. So, a multi-map $\Phi: X \multimap Y$ is coarse if and only if $\Phi$ is $\varphi$-coarse for some $\varphi: \mathcal{E}_{X} \rightarrow \mathcal{E}_{Y}$. It follows that a (single-valued) map $f: X \rightarrow Y$ is coarse if and only if it is coarse as a multi-map.

A multi-map $\Phi: X \multimap Y$ between two balleans is called a coarse embedding if $\Phi^{-1}(Y)=X$ and both maps $\Phi$ and $\Phi^{-1}$ are coarse. If, in addition, $\Phi(X)=Y$, then the multi-map $\Phi: X \multimap Y$ is called a coarse equivalence between the balleans $X$ and $Y$. By analogy with the proof of Proposition 2.1 [3], it can be shown that two balleans $X, Y$ are coarsely equivalent if and only if there is a coarse equivalence $\Phi: X \multimap Y$.

The study of balleans (or coarse spaces) up to their coarse equivalence is one of principal tasks of Coarse Geometry [5], [6], [13], [14].

Example 1.7. Let $G$ be a group. An ideal $\mathcal{I}$ in the Boolean algebra of all subsets of $G$ is called a group ideal if $G=\bigcup \mathcal{I}$ and if for any $A, B \in \mathcal{I}$ we get $A B^{-1} \in \mathcal{I}$.

Let $\mathcal{I}$ be a group ideal $\mathcal{I}$ on a group $G$ and $X$ be a transitive $G$-space endowed with an action $G \times X \rightarrow X$ of the group $G$. The $G$-space $X$ carries the ballean structure $\mathcal{E}_{X, G, \mathcal{I}}=\left\{\varepsilon_{A}\right\}_{A \in \mathcal{I}}$ consisting of the entourages $\varepsilon_{A}=\left\{(x, y) \in X: x \in\left(A \cup\left\{1_{G}\right\} \cup A^{-1}\right) \cdot y\right\}$ parametrized by sets $A \in \mathcal{I}$. Here by $1_{G}$ we denote the unit of the group $G$.

By Theorems 1 and 3 of [7], every (cellular) ballean $\left(X, \mathcal{E}_{X}\right)$ is coarsely isomorphic to the ballean $\left(X, \mathcal{E}_{X, G, \mathcal{I}}\right)$ for a suitable group $G$ of permutations of $X$ and a suitable group ideal $\mathcal{I}$ of $G$ (having a base consisting of subgroups of $G)$.

Example 1.8. Let $G$ be a group endowed with the ballean $\mathcal{E}_{G}$ consisting of entourages $\varepsilon_{F}=\left\{(x, y) \in G \times G: x y^{-1} \in F\right\}$ parametrized by finite subsets $F=F^{-1} \subset G$ containing the unit $1_{G}$ of the group. By [11, 9.8] the ballean $\left(G, \mathcal{E}_{G}\right)$ is cellular if and only if the group $G$ is locally finite (in the sense that each finite subset of $G$ is contained in a finite subgroup of $G)$. By [3], any two infinite countable locally finite groups $G, H$ are coarsely 
equivalent. On the other hand, by [9], two countable locally finite groups $G, H$ are coarsely isomorphic if and only if $\phi_{G}=\phi_{H}$. Here for a group $G$ by $\phi_{G}: \Pi \rightarrow \omega \cup\{\omega\}$ we denote its factorizing function. It is defined on the set $\Pi$ of prime numbers and assigns to each prime number $p \in \Pi$ the (finite or infinite) number

$$
\phi_{G}(p)=\sup \left\{k \in \omega: G \text { contains a subgroup of cardinality } p^{k}\right\} .
$$

\section{A CRITERION FOR A COARSE EQUiVAlenCE OF TWO CELlular ORDINAL BALLEANS}

In this section we introduce two cardinal characteristics called covering numbers of a ballean, and using these cardinal characteristics give a criterion for a coarse equivalence of two cellular ordinal balleans.

Given a subset $A \subset X$ of a set $X$ and an entourage $\varepsilon \subset X \times X$ consider the cardinal

$$
\operatorname{cov}_{\varepsilon}(A)=\min \{|C|: C \subset X, A \subset B(C, \varepsilon)\}
$$

equal to the smallest number of $\varepsilon$-balls covering the set $A$.

For every ballean $\left(X, \mathcal{E}_{X}\right)$ consider the following cardinals:

- $\operatorname{cov}^{\sharp}\left(X, \mathcal{E}_{X}\right)$, equal to the smallest cardinal $\kappa$ for which there is an entourage $\varepsilon \in \mathcal{E}_{X}$ such that for every $\delta \in \mathcal{E}_{X}$ we get

$$
\sup _{x \in X} \operatorname{cov}_{\varepsilon}(B(x, \delta))<\kappa
$$

- $\operatorname{cov}^{b}\left(X, \mathcal{E}_{X}\right)$, equal to the largest cardinal $\kappa$ such that for any cardinal $\lambda<\kappa$ and entourage $\varepsilon \in \mathcal{E}_{X}$ there is $\delta \in \mathcal{E}_{X}$ such that $\min _{x \in X} \operatorname{cov}_{\varepsilon}(B(x, \delta)) \geq \lambda$.

It follows that

$$
\operatorname{cov}^{\sharp}(X)=\min _{\varepsilon \in \mathcal{E}_{X}} \sup _{\delta \in \mathcal{E}_{X}}\left(\sup _{x \in X} \operatorname{cov}_{\varepsilon}(B(x, \delta))\right)^{+}
$$

and

$$
\operatorname{cov}^{b}(X)=\min _{\varepsilon \in \mathcal{E}_{X}} \sup _{\delta \in \mathcal{E}_{X}}\left(\min _{x \in X} \operatorname{cov}_{\varepsilon}(B(x, \delta))\right)^{+},
$$

where $\kappa^{+}$denotes the smallest cardinal which is larger than $\kappa$. Cardinals are identified with the smallest ordinals of given cardinality.

The following proposition can be proved by analogy with the proof of Lemmas 3.1 and 3.2 in [1].

Proposition 2.1. If a ballean $X$ coarsely embeds into a ballean $Y$, then $\operatorname{cov}^{\sharp}(X) \leq \operatorname{cov}^{\sharp}(Y)$. If balleans $X, Y$ are coarsely equivalent, then $\operatorname{cov}^{b}(X)=$ $\operatorname{cov}^{b}(Y)$ and $\operatorname{cov}^{\sharp}(X)=\operatorname{cov}^{\sharp}(Y)$.

Observe that the inequality $\operatorname{cov}^{\sharp}(X) \leq \omega$ means that $X$ has bounded geometry while $\operatorname{cov}^{b}(X) \geq \omega$ means that $X$ has no isolated balls (see [2]). By [3], any two metrizable cellular balleans of bounded geometry and without isolated balls are coarsely equivalent. In [1] this result was extended to 
the following criterion: two metrizable cellular balleans $X, Y$ are coarsely equivalent if $\operatorname{cov}^{b}(X)=\operatorname{cov}^{\sharp}(X)=\operatorname{cov}^{\sharp}(Y)=\operatorname{cov}^{b}(Y)$. In this paper we further extend this criterion to cellular ordinal balleans and prove the following main result of this paper.

Theorem 2.2. Let $X, Y$ be any two cellular ordinal balleans with $\operatorname{cof}(X)=$ $\operatorname{cof}(Y)$.

(1) If $\operatorname{cov}^{\sharp}(X) \leq \operatorname{cov}^{b}(Y)$, then $X$ is coarsely equivalent to a subspace of $Y$.

(2) If $\operatorname{cov}^{b}(X)=\operatorname{cov}^{\sharp}(X)=\operatorname{cov}^{\sharp}(Y)=\operatorname{cov}^{b}(Y)$, then the balleans $X$ and $Y$ are coarsely equivalent.

The proof of this theorem will be presented in Section 6. First we shall discuss some applications of this theorem.

\section{Classifying homogeneous Cellular ordinal Balleans}

In this section we shall apply Theorem 2.2 to show that for a cellular ordinal ballean $X$ the equality $\operatorname{cov}^{b}(X)=\operatorname{cov}^{\sharp}(X)$ is equivalent to the homogeneity of $X$, defined as follows.

A ballean $\left(X, \mathcal{E}_{X}\right)$ is called homogeneous if there is a function $\varphi: \mathcal{E}_{X} \rightarrow \mathcal{E}_{X}$ such that for every points $x, y \in X$ there is a coarse equivalence $\Phi: X \multimap X$ such that $y \in \Phi(x)$ and the multi-maps $\Phi$ and $\Phi^{-1}$ are $\varphi$-coarse. Let us recall that $\Phi: X \multimap X$ is $\varphi$-coarse if $\omega_{\Phi}(\varepsilon):=\bigcup_{(x, y) \in \varepsilon} \Phi(x) \times \Phi(x) \subset \varphi(\varepsilon)$ for every $\varepsilon \in \mathcal{E}_{X}$.

The following proposition shows that homogeneity is preserved by coarse equivalences.

Proposition 3.1. A ballean $X$ is homogeneous if and only if it is coarsely equivalent to a homogeneous ballean $Y$.

Proof. The "only if" part is trivial. To prove the "if" part, assume that a ballean $\left(X, \mathcal{E}_{X}\right)$ admits a coarse equivalence $\Phi: X \multimap Y$ with a homogeneous ballean $\left(Y, \mathcal{E}_{Y}\right)$. By the homogeneity of $\left(Y, \mathcal{E}_{Y}\right)$, there is a function $\varphi_{Y}: \mathcal{E}_{Y} \rightarrow$ $\mathcal{E}_{Y}$ such that for any points $y, y^{\prime} \in Y$ there is a coarse equivalence $\Psi: Y \multimap$ $Y$ such that $y^{\prime} \in \Psi(y)$ and both multi-maps $\Psi$ and $\Psi^{-1}$ are $\varphi_{Y}$-coarse. Since $\Phi$ is a coarse equivalence, there are functions $\varphi_{X, Y}: \mathcal{E}_{X} \rightarrow \mathcal{E}_{Y}$ and $\varphi_{Y, X}: \mathcal{E}_{Y} \rightarrow \mathcal{E}_{X}$ such that $\omega_{\Phi}(\varepsilon) \subset \varphi_{X, Y}(\varepsilon)$ and $\omega_{\Phi^{-1}}(\delta) \subset \varphi_{Y, X}(\delta)$ for every $\varepsilon \in \mathcal{E}_{X}$ and $\delta \in \mathcal{E}_{Y}$. We claim that the function

$$
\varphi_{X}=\varphi_{Y, X} \circ \varphi_{Y} \circ \varphi_{X, Y}: \mathcal{E}_{X} \rightarrow \mathcal{E}_{X}
$$

witnesses that the ballean $X$ is homogeneous. Indeed, given any points $x, x^{\prime}$, we can choose points $y \in \Phi(x), y^{\prime} \in \Phi\left(x^{\prime}\right)$ and find a coarse equivalence $\Psi_{Y}: Y \rightarrow Y$ such that $y^{\prime} \in \Psi_{Y}(y)$ and the multi-maps $\Psi_{Y}$ and $\Psi_{Y}^{-1}$ are $\varphi_{Y^{-}}$ coarse. It can be shown that the multi-map $\Psi_{X}=\Phi^{-1} \circ \Psi_{Y} \circ \Phi: X \multimap X$ has the desired properties: $x^{\prime} \in \Phi^{-1}\left(y^{\prime}\right) \subset \Phi^{-1}\left(\Psi_{Y}(y)\right) \subset \Phi^{-1}\left(\Psi_{Y}(\Phi(x))\right)=$ $\Phi_{X}(x)$ and $\omega_{\Phi_{X}}(\varepsilon) \cup \omega_{\Phi_{X}^{-1}}(\varepsilon) \subset \varphi_{X}(\varepsilon)$ for all $\varepsilon \in \mathcal{E}_{X}$. 
Proposition 3.2. If a ballean $X$ is homogeneous, then $\operatorname{cov}^{b}(X)=\operatorname{cov}^{\sharp}(X)$.

Proof. Since $\operatorname{cov}^{b}(X) \leq \operatorname{cov}^{\sharp}(X)$, it suffices to check that $\operatorname{cov}^{b}(X) \geq \operatorname{cov}^{\sharp}(X)$. This inequality will follow as soon as given $\varepsilon \in \mathcal{E}_{X}$ and a cardinal $\kappa<$ $\operatorname{cov}^{\sharp}(X)$, we find an entourage $\delta \in \mathcal{E}_{X}$ such that $\min _{x \in X} \operatorname{cov}_{\varepsilon}(B(x, \delta)) \geq \kappa$. By the homogeneity of $X$, there is a function $\varphi: \mathcal{E}_{X} \rightarrow \mathcal{E}_{X}$ such that for any points $x, y \in X$ there is a coarse equivalence $\Phi: X \multimap X$ such that $y \in \Phi(x)$ and the multi-maps $\Phi$ and $\Phi^{-1}$ are $\varphi$-coarse.

By the definition of the cardinal $\operatorname{cov}^{\sharp}(X)>\kappa$, for the entourage $\varepsilon^{\prime}=\varphi(\varepsilon)$, there is an entourage $\delta^{\prime} \in \mathcal{E}_{X}$ and a point $x^{\prime} \in X$ such that $\operatorname{cov}_{\mathcal{E}^{\prime}}\left(B\left(x^{\prime}, \delta^{\prime}\right)\right) \geq$ $\kappa$. We claim that the entourage $\delta=\varphi\left(\delta^{\prime}\right) \in \mathcal{E}_{X}$ has the required property: $\min _{x \in X} \operatorname{cov}_{\varepsilon}(B(x, \delta)) \geq \kappa$. Assume conversely that $\operatorname{cov}_{\varepsilon}(B(x, \delta))<\kappa$ for some $x \in X$. By the homogeneity of $X$ and the choice of $\varphi$, there is a coarse equivalence $\Phi: X \multimap X$ such that $x^{\prime} \in \Phi(x)$ and the multi-maps $\Phi$ and $\Phi^{-1}$ are $\varphi$-coarse. Since $\operatorname{cov}_{\varepsilon}(B(x, \delta))<\kappa$, there is a subset $C \subset X$ of cardinality $|C|<\kappa$ such that $B(x, \delta) \subset \bigcup_{c \in C} B(c, \varepsilon)$. For every $c \in C$ fix a point $y_{c} \in \Phi(c)$ and observe that for every point $b \in B(c, \varepsilon)$ we get $(b, c) \in \varepsilon$ and hence $\Phi(b) \times \Phi(c) \subset \omega_{\Phi}(\varepsilon) \subset \varphi(\varepsilon)$ and $\Phi(b) \subset B\left(y_{c}, \varphi(\varepsilon)\right)=B\left(y_{c}, \varepsilon^{\prime}\right)$, which implies $\Phi(B(c, \varepsilon)) \subset B\left(y_{c}, \varepsilon^{\prime}\right)$. Taking into account that $B(x, \delta) \subset$ $\bigcup_{c \in C} B(c, \varepsilon)$, we get $\Phi(B(x, \delta)) \subset \bigcup_{c \in C} \Phi(B(c, \varepsilon)) \subset \bigcup_{c \in C} B\left(y_{c}, \varepsilon^{\prime}\right)$, which implies $\operatorname{cov}_{\varepsilon^{\prime}}(\Phi(B(x, \delta))) \leq|C|<\kappa$.

We claim that $B\left(x^{\prime}, \delta^{\prime}\right) \subset \Phi(B(x, \delta))$. Indeed, for any point $y^{\prime} \in B\left(x^{\prime}, \delta^{\prime}\right)$ we can fix a point $y \in \Phi^{-1}\left(x^{\prime}\right)$ and observe that $\left(y^{\prime}, x^{\prime}\right) \in \delta^{\prime}$ implies $(y, x) \in$ $\Phi^{-1}\left(y^{\prime}\right) \times \Phi^{-1}\left(x^{\prime}\right) \subset \omega_{\Phi^{-1}}\left(\delta^{\prime}\right) \subset \varphi\left(\delta^{\prime}\right)=\delta$ (by the $\varphi$-coarse property of the multi map $\left.\Phi^{-1}\right)$. Then $y \in B(x, \delta)$ and $y^{\prime} \in \Phi(y) \subset \Phi(B(x, \delta))$. Finally, we get $B\left(x^{\prime}, \delta^{\prime}\right) \subset \Phi(B(x, \delta))$ and $\operatorname{cov}_{\mathcal{E}^{\prime}}\left(B\left(x^{\prime}, \delta^{\prime}\right)\right) \leq \operatorname{cov}_{\varepsilon^{\prime}}(\Phi(B(x, \delta))) \leq|C|<$ $\kappa$, which contradicts the choice of $\delta^{\prime}$ and $x^{\prime}$. This completes the proof of the equality $\operatorname{cov}^{b}(X)=\operatorname{cov}^{\sharp}(X)$.

Theorem 3.3. A cellular ordinal ballean $X$ is homogeneous if and only if $\operatorname{cov}^{b}(X)=\operatorname{cov}^{\sharp}(X)$.

Proof. The "only if" part follows from Proposition 3.2. To prove the "if" part, assume $X$ is a cellular ordinal ballean with $\operatorname{cov}^{b}(X)=\operatorname{cov}^{\sharp}(X)$. Let $\gamma=\operatorname{cof}(X)=\operatorname{add}(X)$. The definition of the cardinal $\kappa=\operatorname{cov}^{b}(X)=$ $\operatorname{cov}^{\sharp}(X)$ implies that there exists a non-decreasing transfinite sequence of cardinals $\left(\kappa_{\alpha}\right)_{\alpha<\gamma}$ such that $\kappa=\sup _{\alpha<\gamma} \kappa_{\alpha}^{+}$. Choose an increasing transfinite sequence of groups $\left(G_{\alpha}\right)_{\alpha<\gamma}$ such that $G_{\alpha}=\bigcup_{\beta<\alpha} G_{\beta}$ for every limit ordinal $\alpha<\gamma$ and $\left|G_{\alpha+1} / G_{\alpha}\right|=\kappa_{\alpha}$ for every ordinal $\alpha<\gamma$.

Consider the group $G=\bigcup_{\alpha<\gamma} G_{\alpha}$ endowed with the ballean structure $\mathcal{E}_{G}=\left(\varepsilon_{\alpha}\right)_{\alpha<\gamma}$ consisting of the entourages

$$
\varepsilon_{\alpha}=\left\{(x, y) \in G: x^{-1} y \in G_{\alpha}\right\} \text { for } \alpha<\gamma .
$$

It is clear that the left shifts are id-coarse isomorphisms of $\left(G, \mathcal{E}_{G}\right)$, which implies that the ballean $\left(G, \mathcal{E}_{G}\right)$ is homogeneous. It is clear that add $\left(G, \mathcal{E}_{G}\right)=$ 
$\operatorname{cof}\left(G, \mathcal{E}_{G}\right)=\gamma$ and

$$
\operatorname{cov}^{b}\left(G, \mathcal{E}_{G}\right)=\operatorname{cov}^{\sharp}\left(G, \mathcal{E}_{G}\right)=\min _{\alpha<\gamma} \sup _{\alpha \leq \beta<\gamma}\left|G_{\beta} / G_{\alpha}\right|^{+}=\sup _{\alpha<\gamma} \kappa_{\alpha}^{+}=\kappa .
$$

Applying Theorem 2.2, we conclude that $X$ is coarsely equivalent to the homogeneous ballean $\left(G, \mathcal{E}_{G}\right)$ and hence $X$ is homogeneous according to Proposition 3.1.

The following corollary of Theorems 2.2 and 3.3 shows that the cardinals $\operatorname{cof}(X)$ and $\operatorname{cov}^{\sharp}(X)$ fully determine the coarse structure of a homogeneous cellular ordinal ballean $X$.

Theorem 3.4. For any two homogeneous cellular ordinal balleans $X, Y$ the following conditions are equivalent:

(1) $X$ and $Y$ are coarsely equivalent;

(2) $X$ is coarsely equivalent to a subspace of $Y$ and $Y$ is coarsely equivalent to a subspace of $X$;

(3) $\operatorname{cof}(X)=\operatorname{cof}(Y)$ and $\operatorname{cov}^{\sharp}(X)=\operatorname{cov}^{\sharp}(Y)$.

Proof. The implication (1) $\Rightarrow(2)$ is trivial, the implication $(2) \Rightarrow(3)$ follows by the invariance of the cardinal characteristics cof and cov $^{\sharp}$ under coarse equivalence and their monotonicity under taking subspaces, and the final implication $(3) \Rightarrow(1)$ follows from Theorems 2.2 and 3.3 .

\section{Recognizing the coarse structure of Cantor macro-Cubes AND CARDINAL BALLEANS}

It is easy to see that for any ordinal $\gamma$ and transfinite sequence $\left(\kappa_{\alpha}\right)_{\alpha \in \gamma}$ of non-zero cardinals the asymptotic product $\coprod_{\alpha \in \gamma} \kappa_{\alpha}$ is a homogeneous cellular ordinal ballean whose cofinality equals $\operatorname{cf}(\gamma)$, the cofinality of the ordinal $\gamma$. In particular, the Cantor macro-cube $2^{<\gamma}$ is a homogeneous cellular ordinal ballean of cofinality $\operatorname{cof}\left(2^{<\gamma}\right)=\operatorname{cf}(\gamma)$.

To evaluate the covering numbers of $2^{<\gamma}$, for an ordinal $\gamma$, consider the ordinal

$$
\lfloor\gamma\rfloor=\min \{\alpha: \gamma=\beta+\alpha \text { for some } \beta<\gamma\}
$$

called the tail of $\gamma$, and the cardinal

$$
\lceil\gamma\rceil=\min \{\alpha: \gamma \leq \beta+|\alpha| \text { for some } \beta<\gamma\}
$$

called the cardinal tail of $\gamma$. It is clear that $\lfloor\gamma\rfloor \leq\lceil\gamma\rceil$. Moreover,

$$
\lceil\gamma\rceil= \begin{cases}\lfloor\lfloor\gamma\rfloor \mid & \text { if }\lfloor\gamma\rfloor \text { is a cardinal, } \\ \left\lfloor\left.\lfloor\gamma\rfloor\right|^{+}\right. & \text {otherwise. }\end{cases}
$$

The equality $\gamma=\lfloor\gamma\rfloor$ holds if and only if the ordinal $\gamma$ is additively indecomposable, which means that $\alpha+\beta<\gamma$ for any ordinals $\alpha, \beta<\gamma$.

The following proposition can be derived from the definition of $2^{<\gamma}$. 
Proposition 4.1. For every ordinal $\gamma$ the Cantor macro-cube $2^{<\gamma}$ is a cellular ordinal ballean with

$$
\operatorname{add}\left(2^{<\gamma}\right)=\operatorname{cof}\left(2^{<\gamma}\right)=\operatorname{cf}(\gamma) \text { and } \operatorname{cov}^{b}\left(2^{<\gamma}\right)=\operatorname{cov}^{\sharp}\left(2^{<\gamma}\right)=\lceil\gamma\rceil \text {. }
$$

The following theorem (which can be derived from Proposition 4.1 and Theorem 2.2) shows that in the class of cellular ordinal balleans, the Cantor macro-cubes $2^{<\gamma}$ play a role analogous to the role of the Cantor cubes $2^{\kappa}$ in the class of zero-dimensional compact Hausdorff spaces.

Theorem 4.2. Let $\gamma$ be any ordinal and $X$ be any cellular ordinal ballean such that $\operatorname{cof}(X)=\operatorname{cf}(\gamma)$.

(1) If $\lceil\gamma\rceil \leq \operatorname{cov}^{b}(X)$, then $2^{<\gamma}$ is coarsely equivalent to a subspace of $X$

(2) If $\operatorname{cov}^{\sharp}(X) \leq\lceil\gamma\rceil$, then $X$ is coarsely equivalent to a subspace of $2^{<\gamma}$.

(3) If $\operatorname{cov}^{b}(X)=\operatorname{cov}^{\sharp}(X)=\lceil\gamma\rceil$, then $X$ is coarsely equivalent to $2^{<\gamma}$.

Proposition 4.1 and Theorem 4.2 imply the following characterization of the Cantor macro-cube $2^{<\gamma}$ which extends the characterization of the Cantor macro-cube $2^{<\omega}$ proved in [3].

Theorem 4.3. For any ordinal $\gamma$ and any ballean $X$ the following conditions are equivalent:

(1) $X$ is coarsely equivalent to $2^{<\gamma}$;

(2) $X$ is cellular, $\operatorname{add}(X)=\operatorname{cof}(X)=\operatorname{cf}(\gamma)$ and $\operatorname{cov}^{b}(X)=\operatorname{cov}^{\sharp}(X)=$ $\lceil\gamma\rceil$.

Corollary 4.4. For any two ordinals $\beta, \gamma$ the Cantor macro-cubes $2^{<\beta}$ and $2^{<\gamma}$ are coarsely equivalent of and only if $\operatorname{cf}(\beta)=\operatorname{cf}(\gamma)$ and $\lceil\beta\rceil=\lceil\gamma\rceil$.

Finally, we recognize the coarse structure on the ballean $\stackrel{\leftrightarrow}{\gamma}$ supported by an additively indecomposable ordinal $\gamma$. Given any non-zero ordinal $\gamma$ we consider the family $\left\{\varepsilon_{\alpha}\right\}_{\alpha<\gamma}$ of the entourages

$$
\varepsilon_{\alpha}=\{(x, y) \in \gamma \times \gamma: x \leq y+\alpha \text { and } y \leq x+\alpha\}
$$

for $\alpha<\gamma$. It is easy to see that $\left(\gamma,\left\{\varepsilon_{\alpha}\right\}\right)_{\alpha<\gamma}$ is a ballean if and only if the ordinal $\gamma$ is additively indecomposable (which means that $\alpha+\beta<\gamma$ for any ordinals $\alpha, \beta<\gamma)$. This ballean will be denoted by $\overleftrightarrow{\gamma}$.

The following theorem classifies the balleans $\overleftrightarrow{\gamma}$ up to coarse equivalence.

Theorem 4.5. For any additively indecomposable ordinal $\gamma$ the ballean $\overleftrightarrow{\gamma}$ is coarsely equivalent to:

- $\overleftrightarrow{\omega}$ if and only if $\gamma=\beta \cdot \omega$ for some $\beta$

- $2^{<\gamma}$, otherwise.

Proof. If $\gamma=\beta \cdot \omega$ for some ordinal $\beta$, then the ballean $\overleftrightarrow{\gamma}$ is coarsely equivalent to $\overleftrightarrow{\omega}$ since $\stackrel{\leftrightarrow}{\gamma}$ contains the large subset $L=\{\beta \cdot n: n \in \omega\}$, which is coarsely isomorphic to $\overleftrightarrow{\omega}$. 
Now assume that $\gamma \neq \beta \cdot \omega$ for any ordinal $\beta$. Since $\gamma$ is additively indecomposable, this means that $\beta \cdot \omega<\gamma$ for any ordinal $\beta<\gamma$, which implies that the ballean $\overleftrightarrow{\gamma}$ is cellular. Since $\operatorname{add}(\stackrel{\leftrightarrow}{\gamma})=\operatorname{cof}(\overleftrightarrow{\gamma})=\operatorname{cf}(\gamma)$ and $\operatorname{cov}^{b}(\overleftrightarrow{\gamma})=\operatorname{cov}^{\sharp}(\overleftrightarrow{\gamma})=\lceil\gamma\rceil$, the cellular ordinal ballean $\stackrel{\leftrightarrow}{\gamma}$ is coarsely equivalent to $2^{<\gamma}$ according to Theorem 4.3.

Remark 4.6. Let us observe that for any ordinal $\gamma$ the balleans $2^{<\gamma}$ and $\overleftrightarrow{\omega}$ are not coarsely equivalent since the ballean $2^{<\gamma}$ is cellular whereas $\overleftrightarrow{\omega}$ is not.

\section{EMBEDDING CELLULAR ORDINAL BALLEANS INTO ASYMPTOTIC PRODUCTS OF CARDINALS}

In this section we construct coarse embeddings of cellular ordinal balleans into asymptotic products of cardinals. This embedding will play a crucial role in the proof of Theorem 2.2 presented in the next section.

Let us observe that for any transfinite sequence of cardinals $\left(\kappa_{\alpha}\right)_{\alpha<\gamma}$ the asymptotic product $\coprod_{\alpha<\gamma} \kappa_{\alpha}$ carries an operation of coordinatewise addition of sequences induces by the operation of addition of ordinals. For ordinals $\beta<\gamma$ and $y \in \kappa_{\alpha}$ let $y \cdot \delta_{\beta}$ denote the sequence $\left(x_{\alpha}\right)_{\alpha<\gamma} \in \coprod_{\alpha<\gamma} \kappa_{\alpha}$ such that $x_{\alpha}=y$ if $\alpha=\beta$ and $x_{\alpha}=0$ otherwise. It follows that each element $\left(x_{\alpha}\right)_{\alpha<\gamma} \in \coprod_{\alpha<\gamma} \kappa_{\alpha}$ can be written as $\sum_{\alpha \in A} x_{\alpha} \cdot \delta_{\alpha}$ for the finite set $A=$ $\left\{\alpha<\gamma: x_{\alpha} \neq 0\right\}$.

The following lemma exploits and develops the decomposition technique used in [9], [11, §10] and [12].

Lemma 5.1. Let $X$ be an ordinal ballean of infinite cofinality $\gamma$ and $\left(\varepsilon_{\alpha}\right)_{\alpha<\gamma}$ be a well-ordered base of the coarse structure of $X$ consisting of cellular entourages such that $\varepsilon_{\beta}=\bigcup_{\alpha<\beta} \varepsilon_{\alpha}$ for all limit ordinals $\beta<\gamma$. For every $\alpha<\gamma$ and $x \in X$ let $\kappa_{\alpha}(x)=\operatorname{cov}_{\varepsilon_{\alpha}}\left(B\left(x, \varepsilon_{\alpha+1}\right)\right)$ and put $\kappa_{\alpha}=\min _{x \in X} \kappa_{\alpha}(x)$ and $\bar{\kappa}_{\alpha}=\sup _{x \in X} \kappa_{\alpha}(x)$. Then the ballean $X$ is coarsely equivalent to $a$ subbalean $Y \subset \coprod_{\alpha<\gamma} \bar{\kappa}_{\alpha}$ containing the set $\coprod_{\alpha<\lambda} \kappa_{\alpha}$.

Proof. For each two points $x, y \in X$ let

$$
d(x, y)=\min \left\{\alpha<\gamma:(x, y) \in \varepsilon_{\alpha}\right\}
$$

and observe that for any pair $(x, y) \notin \varepsilon_{0}$ the ordinal $d(x, y)$ is not limit (as $\varepsilon_{\beta}=\bigcup_{\alpha<\beta} \varepsilon_{\alpha}$ for any limit ordinal $\left.\beta<\gamma\right)$. Consequently we can find an ordinal $d^{-}(x, y)$ such that $d(x, y)=d^{-}(x, y)+1$.

Fix any well-ordering $\preceq$ of the set $X$. Given a non-empty subset $B \subset X$ denote by $\min B$ the smallest point of $B$ with respect to the well-order $\preceq$ and for every $\alpha<\gamma$ let $c_{\alpha}: X \rightarrow X$ be the map assigning to each point $x \in X$ the smallest element $c_{\alpha}(x)=\min B\left(x, \varepsilon_{\alpha}\right)$ of the ball $B\left(x, \varepsilon_{\alpha}\right)$. Since $\varepsilon_{\alpha}$ is an equivalence relation, $B\left(x, \varepsilon_{\alpha}\right)=B\left(c_{\alpha}(x), \varepsilon_{\alpha}\right)$. To simplify the notation in the sequel we shall denote the ball $B\left(x, \varepsilon_{\alpha}\right)$ by $B_{\alpha}(x)$.

Observe that for every $\alpha<\gamma$ and ball $B \in\left\{B_{\alpha+1}(x): x \in X\right\}$ the set $c_{\alpha}(X) \cap B$ has cardinality $\kappa_{\alpha}(\min B)$, so we can fix a map $n_{\alpha, B}: B \rightarrow$ 
$\kappa_{\alpha}(\min B)$ such that $\left\{B_{\alpha}(x): x \in B\right\}=\left\{n_{\alpha, B}^{-1}(\beta): \beta \in \kappa_{\alpha}(\min B)\right\}$ and $n_{\alpha, B}^{-1}(0)=B_{\alpha}(\min B)$. Finally, define a map $n_{\alpha}: X \rightarrow \bar{\kappa}_{\alpha}$ assigning to each point $y \in X$ the number $n_{\alpha}(y):=n_{\alpha, B_{\alpha+1}(y)}(y)$ of the $\varepsilon_{\alpha}$-ball containing $y$ in the partition of the $\varepsilon_{\alpha+1}$-ball $B_{\alpha+1}(y)$. The definition of the cardinal $\kappa_{\alpha}$ implies that $\kappa_{\alpha} \subset \kappa_{\alpha}(x)=n_{\alpha}\left(B_{\alpha+1}(x)\right)$ for every $x \in X$.

For every $x \in X$ define a map $f_{x}: X \rightarrow \coprod_{\alpha<\gamma} \bar{\kappa}_{\alpha}$ by the recursive formula

$$
f_{x}(y)= \begin{cases}0 & \text { if } d(x, y)=0 \\ f_{\min B_{d^{-}(x, y)}(y)}(y)+n_{d^{-}(x, y)}(y) \cdot \delta_{d^{-}(x, y)} & \text { otherwise. }\end{cases}
$$

Since $d\left(\min B_{d^{-}(x, y)}(y), y\right)<d(x, y)$ the function $f_{x}$ is well-defined.

It can be shown that for every $x \in X$ the function $f_{x}: X \rightarrow \coprod_{\alpha<\gamma} \bar{\kappa}_{\alpha}$ determines a coarse equivalence of $X$ with the subspace $f(X)$ of $\coprod_{\alpha<\gamma} \bar{\kappa}_{\alpha}$ containing the asymptotic product $\coprod_{\alpha<\gamma} \kappa_{\alpha}$.

\section{Proof of Theorem 2.2}

Assume that $X, Y$ are two cellular balleans with $\gamma=\operatorname{add}(X)=\operatorname{cof}(X)=$ $\operatorname{cof}(Y)=\operatorname{add}(X)$ and $\kappa=\operatorname{cov}^{b}(X)=\operatorname{cov}^{\sharp}(X)=\operatorname{cov}^{\sharp}(Y)=\operatorname{cov}^{b}(Y)$ for some cardinals $\gamma$ and $\kappa$. Let $\mathcal{E}_{X}, \mathcal{E}_{Y}$ denote the ballean structures of $X$ and $Y$, respectively.

Separately we shall consider 4 cases.

1) $\gamma=0$. In this case the balleans $X, Y$ are empty and hence coarsely equivalent.

2) $\gamma=1$. In this case the balleans $X, Y$ are bounded and hence are coarsely equivalent.

3) $\gamma=\omega$. Since $X$ is a cellular ballean with $\operatorname{cof}(X)=\gamma=\omega$, the coarse structure $\downarrow \mathcal{E}_{X}$ of $X$ has a well-ordered base $\left\{\varepsilon_{n}\right\}_{n \in \omega}$ consisting of equivalence relations such that $\varepsilon_{0}=\Delta_{X}$. In this case the formula

$$
d_{X}\left(x, x^{\prime}\right)=\min \left\{n \in \omega:\left(x, x^{\prime}\right) \in \varepsilon_{n}\right\}
$$

defines an ultrametric $d_{X}: X \times X \rightarrow \omega$ generating the coarse structure of the ballean $X$. By analogy we can define an ultrametric $d_{Y}$ generating the coarse structure of the ballean $Y$. Since $\operatorname{cov}^{b}(X)=\operatorname{cov}^{\sharp}(X)=\operatorname{cov}^{\sharp}(Y)=\operatorname{cov}^{b}(Y)$, we can apply Theorem 1.2 of [1] (proved by the technique of towers created in [3]) to conclude that the ultrametric spaces $X$ and $Y$ are coarsely equivalent.

4) $\gamma>\omega$. Since $X, Y$ are ordinal balleans of cofinality $\operatorname{cof}(X)=\operatorname{cof}(Y)=$ $\gamma$, we can fix well-ordered bases $\left\{\tilde{\varepsilon}_{\alpha}\right\}_{\alpha<\gamma}$ and $\left\{\tilde{\delta}_{\alpha}\right\}_{\alpha<\gamma}$ of the coarse structures $\downarrow \mathcal{E}_{X}$ and $\downarrow \mathcal{E}_{Y}$, respectively.

By induction on $\alpha<\gamma$ we shall construct well-ordered sequences $\left\{\varepsilon_{\alpha}\right\}_{\alpha<\gamma} \subset$ $\downarrow \mathcal{E}_{X}$ and $\left\{\delta_{\alpha}\right\}_{\alpha<\gamma} \subset \downarrow \mathcal{E}_{Y}$ such that for every $\alpha<\gamma$ the following conditions will be satisfied:

(a) $\varepsilon_{\alpha}=\bigcup_{\beta<\alpha} \varepsilon_{\beta}$ and $\delta_{\alpha}=\bigcup_{\beta<\alpha} \delta_{\beta}$ if the ordinal $\alpha$ is limit;

(b) $\varepsilon_{\alpha}$ and $\delta_{\alpha}$ are cellular entourages; 
(c) $\tilde{\varepsilon}_{\alpha} \subset \varepsilon_{\alpha+1}$ and $\tilde{\delta}_{\alpha} \subset \delta_{\alpha+1}$;

(d) for some cardinal $\kappa_{\alpha}, \min _{x \in X} \operatorname{cov}_{\varepsilon_{\alpha}}\left(B\left(x, \varepsilon_{\alpha+1}\right)\right)=\sup _{x \in X} \operatorname{cov}_{\varepsilon_{\alpha}}\left(B\left(x, \varepsilon_{\alpha+1}\right)\right)=$ $\min _{y \in Y} \operatorname{cov}_{\delta_{\alpha}}\left(B\left(y, \delta_{\alpha+1}\right)\right)=\sup _{y \in Y} \operatorname{cov}_{\delta_{\alpha}}\left(B\left(y, \delta_{\alpha+1}\right)\right)=\kappa_{\alpha}$.

We start the inductive construction by choosing cellular entourages $\varepsilon_{0} \in$ $\mathcal{E}_{X}$ and $\delta_{0} \in \mathcal{E}_{Y}$ such that

$$
\sup _{x \in X} \operatorname{cov}_{\varepsilon_{0}}(B(x, \varepsilon))<\kappa \text { and } \sup _{y \in Y} \operatorname{cov}_{\delta_{0}}(B(y, \delta))<\kappa
$$

for any entourages $\varepsilon \in \downarrow \mathcal{E}_{X}$ and $\delta \in \downarrow \mathcal{E}_{Y}$. The existence of such entourages $\varepsilon_{0}$ and $\delta_{0}$ follows from the cellularity of $X, Y$ and the definition of the cardinals $\operatorname{cov}^{\sharp}(X)=\operatorname{cov}^{\sharp}(Y)=\kappa$. Assume that for some ordinal $\alpha<\gamma$ and all ordinals $\beta<\alpha$ the cellular entourages $\varepsilon_{\beta}$ and $\delta_{\beta}$ have already been constructed. If the ordinal $\alpha$ is limit, then we put $\varepsilon_{\alpha}=\bigcup_{\beta<\alpha} \varepsilon_{\beta}$ and $\delta_{\alpha}=\bigcup_{\beta<\alpha} \delta_{\beta}$ and observe that the entourages $\varepsilon_{\alpha}$ and $\delta_{\beta}$ are cellular as unions of increasing chains of cellular entourages. Moreover, $\varepsilon_{\alpha} \in \downarrow \mathcal{E}_{X}$ and $\delta_{\beta} \in \downarrow \mathcal{E}_{Y}$ as $\alpha<\gamma=\operatorname{add}(X)=\operatorname{add}(Y)$.

Next, assume that the ordinal $\alpha$ is not limit and hence $\alpha=\beta+1$ for some ordinal $\beta$. Taking into account the choice of the entourages $\varepsilon_{0}, \delta_{0}$ and using the definitions of the cardinals $\operatorname{cov}^{b}(X)=\operatorname{cov}^{b}(Y)$, we can construct two increasing sequences of cellular entourages $\left\{\varepsilon_{n}^{\prime}\right\}_{n \in \omega} \subset \downarrow \mathcal{E}_{X}$ and $\left\{\delta_{n}^{\prime}\right\}_{n \in \omega} \subset$ $\downarrow \mathcal{E}_{Y}$ such that

$$
\sup _{x \in X} \operatorname{cov}_{\varepsilon_{n}^{\prime}}\left(B\left(x, \varepsilon_{n+1}^{\prime}\right)\right) \leq \min _{y \in Y} \operatorname{cov}_{\delta_{n}^{\prime}}\left(B\left(y, \delta_{n+1}^{\prime}\right)\right)
$$

and

$$
\sup _{y \in Y} \operatorname{cov}_{\delta_{n}^{\prime}}\left(B\left(y, \delta_{n+1}^{\prime}\right)\right) \leq \min _{x \in X} \operatorname{cov}_{\varepsilon_{n+1}^{\prime}}\left(B\left(x, \varepsilon_{n+2}^{\prime}\right)\right) .
$$

The entourages $\varepsilon_{1}^{\prime}$ and $\delta_{1}^{\prime}$ can be chosen so that $\tilde{\varepsilon}_{\alpha} \subset \varepsilon_{1}^{\prime}$ and $\tilde{\delta}_{\alpha} \subset \delta_{1}^{\prime}$. Since $\operatorname{add}(X)=\operatorname{add}(Y)>\omega$, the entourages $\varepsilon_{\alpha+1}=\bigcup_{n \in \omega} \varepsilon_{n}^{\prime}$ and $\delta_{\alpha+1}=\bigcup_{n \in \omega} \delta_{n}^{\prime}$ belong to the coarse structures $\downarrow \mathcal{E}_{X}$ and $\downarrow \mathcal{E}_{Y}$, respectively, and have the properties (b)-(d), required in the inductive construction.

By Lemma 5.1, there are coarse equivalences $f_{X}: X \rightarrow \coprod_{\alpha<\gamma} \kappa_{\alpha}$ and $f_{Y}: Y \rightarrow \coprod_{\alpha<\gamma} \kappa_{\alpha}$. Then the multi-map $f_{Y}^{-1} \circ f_{X}: X \multimap Y$ is a coarse equivalence between the balleans $X$ and $Y$.

\section{ACKnowledgements}

This research was supported by the Slovenian Research Agency program P1-0292. 


\section{REFERENCES}

[1] T. Banakh, D. Repovš, Classifying homogeneous ultrametric spaces up to coarse equivalence, Colloq. Math. 144:2 (2016), 189-202.

[2] T. Banakh, I. Zarichnyi, A coarse characterization of the Baire macro-space, Proc. of Intern. Geometry Center. 3:4 (2010) 6-14 (available http://arxiv.org/abs/1103.5118).

[3] T. Banakh, I. Zarichnyi, Characterizing the Cantor bi-cube in asymptotic categories, Groups Geom. Dyn. 5:4 (2011), 691-728.

[4] N. Brodsky, J. Dydak, J. Higes, A. Mitra, Dimension zero at all scales, Topology Appl. 154:14 (2007), 2729-2740.

[5] S. Buyalo, V. Schroeder, Elements of Asymptotic Geometry, EMS Monographs in Mathematics. (EMS), Zürich, 2007.

[6] P. Nowak, G. Yu, Large Scale Geometry, EMS Textbooks in Mathematics. (EMS), Zürich, 2012.

[7] O. Petrenko, I.V. Protasov, Balleans and G-spaces, Ukr. Math J. 64 (2012), 344-350.

[8] O. Petrenko, I.V. Protasov, S. Slobodianiuk, Asymptotic structures of cardinals, Appl. Gen. Topology, 15:2 (2014), 137-146.

[9] I.V. Protasov, Morphisms of balls structures of groups and graphs, Ukr. Mat. Zh. 54:6 (2002), 847-855.

[10] I.V. Protasov, Normal ball structures, Math. Stud. 20 (2003), 3-16.

[11] I. Protasov, T. Banakh, Ball stuctures and colorings of graphs and groups, VNTL Publ. 2003, 148p.

[12] I.V. Protasov, A. Tsvietkova, Decomposition of cellular balleans, Top. Proc. 36 (2010), $77-83$.

[13] I. Protasov M. Zarichnyi, General Asymptology, Monograph Series, Vol.12, VNTL Publ., Lviv, 2007.

[14] J. Roe, Lectures on Coarse Geometry, Univ. Lecture Series, Vol. 31, Amer. Math. Soc., 2003.

T. Banakh: Ivan Franko National University, Lviv, Ukraine \& Jan Kochanowski University, Kielce, Poland

E-mail address: t.o.banakh@gmail.com

I. Protasov: Faculty of Cybernetics, Taras Shevchenko National UniverSity, Kiev, Ukraine

E-mail address: i.v.protasov@gmail.com

D. Repovš: Faculty of Education, and Faculty of Mathematics and Physics, University of Ludbljana \& Institute of Mathematics, Physics and Mechanics, LuUbluana, Slovenia

E-mail address: dusan.repovs@guest.arnes.si

S. Slobodianiuk: Faculty of Cybernetics, Taras Shevchenko National UniVERSITY, KIEV, UKRAINE

E-mail address: slobodianiuk@yandex.ru 